\title{
Design, Synthesis, and Testing of a Molecular Truck for Colonic Delivery of 5-Aminosalicylic Acid
}

Suryakiran Navath, ${ }^{\dagger}$ Venkataramanarao Rao, ${ }^{\dagger}$ Rita-Marie T. Woodford, ${ }^{\ddagger},{ }^{\S}$ Monica T. Midura-Kiela, ${ }^{\ddagger}$ Ali M. Ahad, ${ }^{\dagger}$ Ramesh Alleti, ${ }^{\dagger}$ Pawel R. Kiela, ${ }^{*},+, \|$ and Eugene A. Mash ${ }^{*}, \dagger$

${ }^{\dagger}$ Department of Chemistry and Biochemistry, University of Arizona, Tucson, Arizona 85721, United States

${ }^{\ddagger}$ Department of Pediatrics, University of Arizona, Tucson, Arizona 85724, United States

${ }^{\S}$ School of Dentistry, Oral Biology Program, University of North Carolina, Chapel Hill, North Carolina 27599, United States

"Department of Immunobiology, University of Arizona, Tucson, Arizona 85724, United States

\section{Supporting Information}

\begin{abstract}
A molecular scaffold bearing eight terminal alkyne groups was synthesized from sucrose. Eight copies of an azide-terminated, azolinked precursor to 5-aminosalicylic acid were attached to the scaffold via copper(I)-catalyzed azide-alkyne cycloaddition. The resulting compound was evaluated in a DSS model of colitis in BALB/c mice against sulfasalazine as a control. Two independent studies verified that the novel pro-drug, administered in a dose calculated to result in an equimolar 5ASA yield, outperformed sulfasalazine in terms of protection from mucosal inflammation and $\mathrm{T}$ cell activation. A separate study established that 5ASA appeared in feces produced 24-48 h following administration of the pro-drug. Thus, a new, orally administered pro-drug form of 5-aminosalicylic acid has been developed and successfully demonstrated.

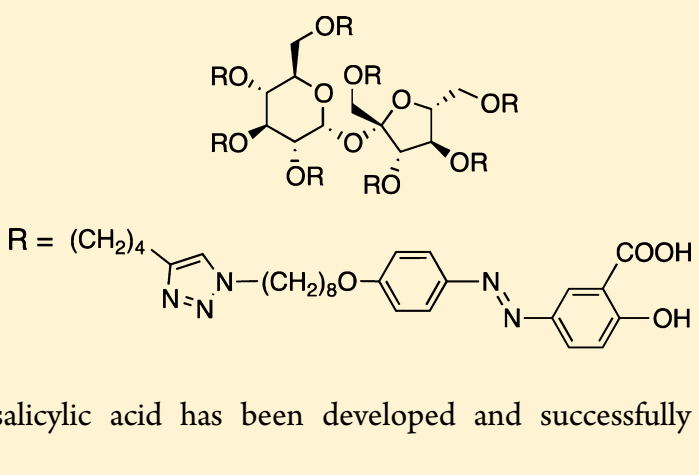

KEYWORDS: Inflammatory bowel disease, 5-aminosalicylic acid, pro-drug

$\mathrm{M}$ esalamine (mesalazine, 5-aminosalicylic acid, 5-ASA, 1) remains the compound of choice for the treatment of inflammatory bowel disease (IBD) ${ }^{1-3}$ 5-ASA is used to induce remission and/or to prevent relapses, and as a means of prevention of IBD-associated colon cancer. Among its known mechanisms of action are inhibition of IL- $1 \beta$ and $\mathrm{TNF} \alpha$ signaling, ${ }^{4-6}$ downstream inhibition of NF- $\kappa \mathrm{B},{ }^{7,8}$ and activation of peroxisome proliferator-activated receptors in colonic epithelial cells, which are involved in the control of inflammation, cell proliferation, apoptosis, and metabolic function. ${ }^{9}$

Colon-specific delivery of 5-ASA can be achieved by the use of enemas and suppositories. However, when administered orally, rapid absorption of 5-ASA through the mucosa of the upper gastrointestinal (GI) tract greatly reduces its bioavailability to the colonic mucosa. ${ }^{9}$ Approaches to enhancing colonspecific delivery of orally administered 5-ASA include the use of $\mathrm{pH}$ sensitive polymer coatings, ${ }^{10}$ biodegradable polymer matrices and hydrogels, ${ }^{11}$ and pro-drugs. In this last category, bioactivation by reductive cleavage of azo links by bacterial azoreductases in the gut is exploited in the case of the prototypical 5-ASA pro-drug, sulfasalazine (2). ${ }^{12}$ Concomitant release of the carrier, sulfapyridine (3), is believed to be responsible for a number of side effects. ${ }^{13}$ The related prodrugs olsalazine (Dipentum) and balsalazide (Colazal) depend on a similar 5-ASA release strategy.

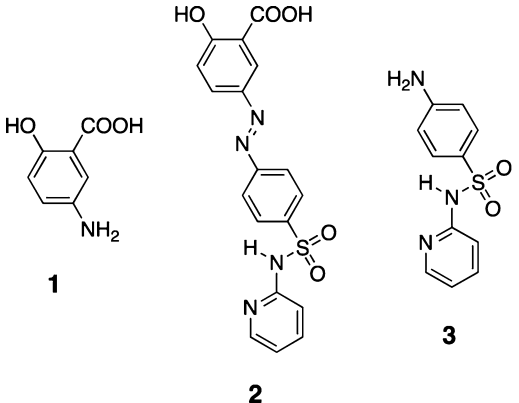

In designing a new 5-ASA carrier and pro-drug, we set the following objectives: (1) the pro-drug should be administered orally and (2) deliver a highly concentrated payload to the appropriate region of the gastrointestinal tract; (3) the carrier should be excreted in the feces with little or no absorption or breakdown, and (4) have negligible, if any, side effects. With these goals in mind, the properties of Olestra, a type of "fake fat" derived by esterification of sucrose with a mixture of fatty acids, drew our attention. Olestra passes, unmodified and unabsorbed, through the entire GI tract. ${ }^{14-16}$ This suggested that a related compound, with metabolism and disposition similar to that of Olestra, but with fewer side effects owing to

Received: April 6, 2012

Accepted: July 29, 2012

Published: August 1, 2012 
Scheme 1. Synthesis of Azide $9^{a}$

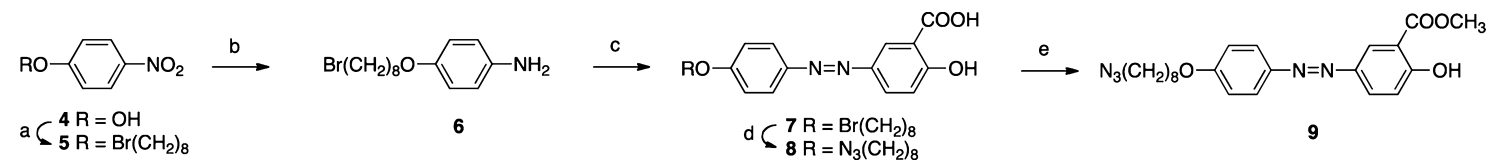

${ }^{a}$ Reagents and conditions: (a) $\mathrm{K}_{2} \mathrm{CO}_{3}$, 1,8-dibromooctane, acetone; (b) $10 \% \mathrm{Pd} / \mathrm{C}, \mathrm{H}_{2}$ (40 psi), EtOH; (c) $\mathrm{NaNO}_{2}$, aq $\mathrm{HCl}_{\text {; }}$ salicylic acid, aq $\mathrm{NaOH}$; (d) $\mathrm{NaN}_{3}$, DMSO; (e) $\mathrm{K}_{2} \mathrm{CO}_{3},\left(\mathrm{CH}_{3}\right)_{2} \mathrm{SO}_{4}$, acetone.

Scheme 2. Synthesis of Octatriazole Sodium Salt $13^{a}$
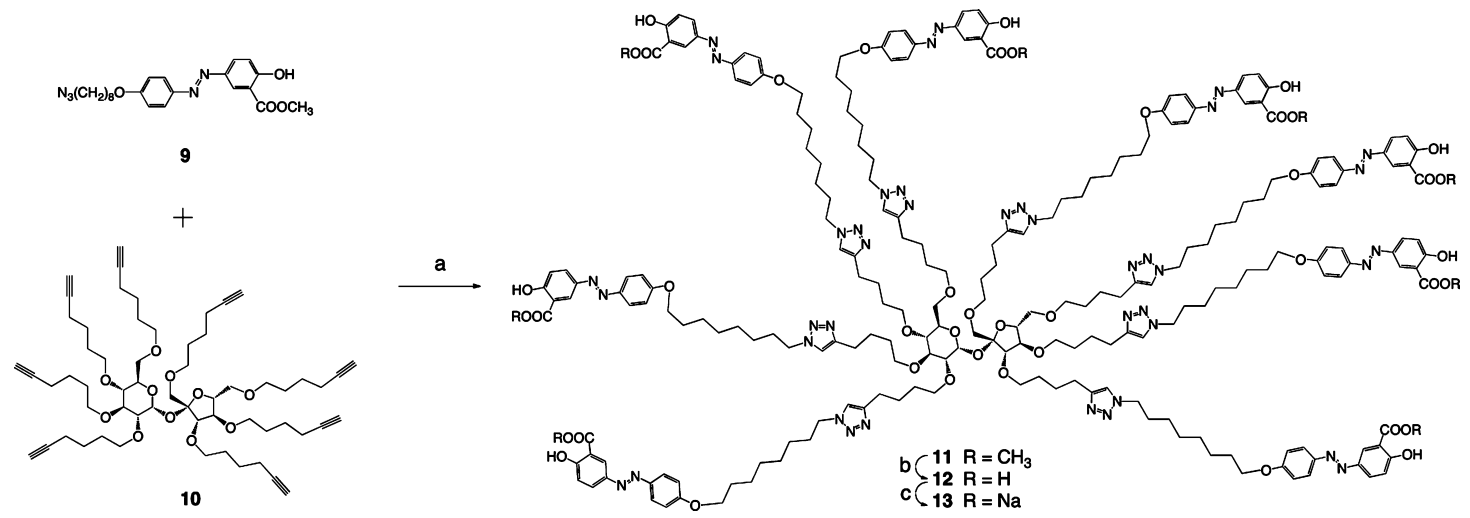

${ }^{a}$ Reagents and conditions: (a) $\mathrm{CuSO}_{4}$, sodium ascorbate, THF/water; (b) LiOH, THF/water/methanol; (c) ion exchange chromatography.

structural changes and/or reduction in the administered dose, would be an excellent candidate for use as a drug transport scaffold in the GI system. For treatment of IBD, a branched structure would allow attachment of multiple 5-ASA precursors to the scaffold, producing a high payload pro-drug that might provide a means for colon-specific delivery, achieve higher concentrations of the active drug, and lead to improved healing.

Olestra contains acid-labile and base-labile ester links and is a complex mixture of compounds. To make our scaffold more stable and less complex, we elected to employ ether links to sucrose involving chains of the same constitution. Our recent work on construction of multivalent compounds ${ }^{17-19}$ suggested the use of copper(I)-catalyzed azide-alkyne cycloaddition (CuAAC $)^{20,21}$ for attachment of the 5-ASA precursors to the scaffold. We report herein the design, synthesis, and biological testing of a sucrose-derived "molecular truck" loaded with eight copies of 5-ASA in a form releasable by bacterial azoreductases in the gut.

The synthesis of 5-ASA derivative 9 is depicted in Scheme 1. Reaction of $p$-nitrophenol (4) with potassium carbonate and 5 equiv of 1,8-dibromooctane in acetone gave ether 5 in $89 \%$ yield. $^{22}$ Nitro reduction was effected by hydrogenation using $10 \% \mathrm{Pd} / \mathrm{C}$ catalyst, producing aniline 6 in 93\% yield. Treatment of $\mathbf{6}$ with sodium nitrite in aqueous hydrochloric acid gave the corresponding diazonium salt, which was allowed to react with salicylic acid in an aqueous alkaline solution. This gave the azobenzene derivative 7 in $40 \%$ yield. Reaction of bromide 7 with sodium azide in DMSO produced the corresponding azide $\mathbf{8}$ in $91 \%$ yield. Treatment of $\mathbf{8}$ with potassium carbonate and dimethyl sulfate in refluxing acetone gave ester 9 in $50 \%$ yield.

$\mathrm{CuAAC}$ reaction ${ }^{20,21}$ of sucrose-derived octaalkyne $10^{19}$ with 10 equiv of azide 9 using $\mathrm{CuSO}_{4}$ and sodium ascorbate in deoxygenated (with argon) 9/1 THF/water at room temperature for $12 \mathrm{~h}$ produced, after column chromatography, octatriazole octaester 11 in $68 \%$ yield (Scheme 2). The structure of 11 was confirmed by NMR and MALDI-TOF MS analyses (see Supporting Information). ${ }^{23}$

Saponification of 11 using $\mathrm{LiOH}$ in 9/3.3/1 THF/water/ methanol gave acid 12, which was converted to the sodium salt 13 by ion exchange chromatography and recovered from solution by lyophilization. Compound 13 was a nonhydroscopic bright yellow solid that was characterized by ${ }^{1} \mathrm{H}$ NMR and MALDI-TOF analysis (see Supporting Information).

An animal model was utilized to compare the efficacy of 13 in relation to 2 , a medicine used in treatment of IBD. For this in vivo model, 8-10 week-old male BALB/c mice were given a 5 day treatment of drinking water containing $4 \%$ dextran sulfate sodium salt (DSS), which causes damage to and inflammation of the epithelial lining of the colon. Mice were then given regular drinking water and were monitored for a total of 12 days. During the study, mice were given daily gastric gavage feedings of (a) placebo suspension medium (Ora-Blend SF, Paddock Laboratories), (b) suspension medium containing 2, or (c) suspension medium containing 13. A dose of $0.28 \mathrm{mmol}$ of 5-ASA equivalent/( $\mathrm{kg}$ day) was chosen based on published literature. ${ }^{24-26}$ Thus, the amounts of $2(111.5 \mathrm{mg} /(\mathrm{kg}$ day $))$ and $13(155.7 \mathrm{mg} /(\mathrm{kg}$ day $))$ were calculated to give the same theoretical yield of 5-ASA after azo reduction, assuming 100\% efficiency. Control groups included mice given regular drinking water and placebo, as well as mice treated with DSS and placebo.

The results of the first study demonstrated decreased morbidity and mortality for mice treated with 13 when compared to mice treated with either DSS and placebo or DSS and 2 (Figure 1). In general, mice treated with 13 had a much better appearance and displayed significantly less behavioral depression, as evidenced by posture, mobility, grooming, and social behavior (data not shown). Mice treated with 13 exhibited less weight loss (Figure 1A) and colon shortening (Figure 1B) as a measure of inflammation. Survival (Figure 1C) was equal among mice treated with 13 and untreated control 

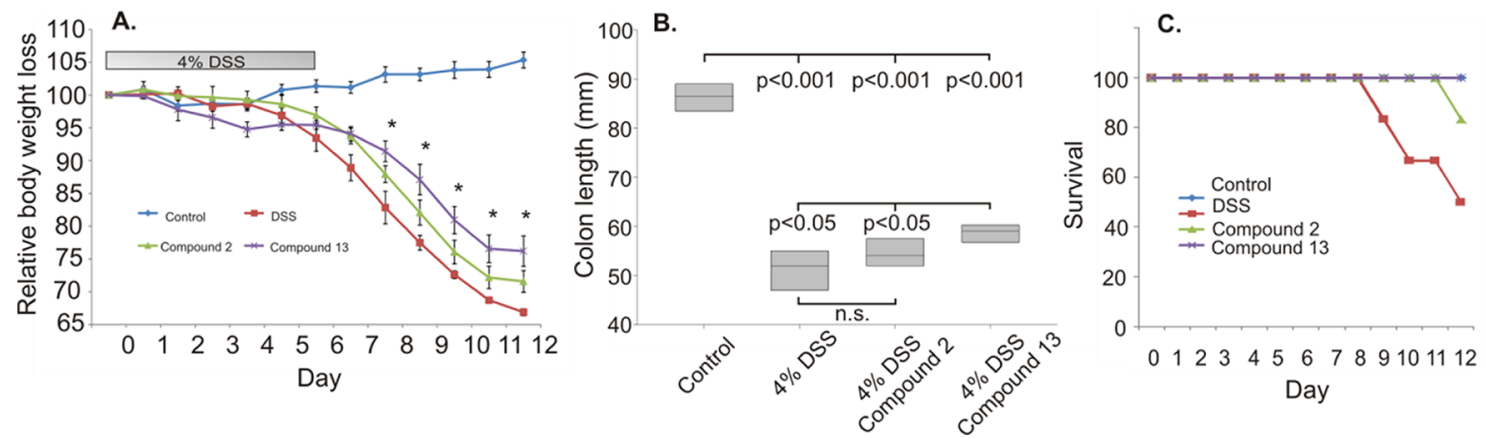

Figure 1. (A) Body weight loss in 4\% DSS colitis. Data were analyzed statistically with AVOVA followed by a Fisher PLSD posthoc test. * differences between DSS and DSS +2 or DSS +13 at day 7, and between DSS +13 and DSS or DSS +2 at days 8-12. (B) Changes in colon length in DSS colitis. Data were analyzed statistically with AVOVA followed by a Fisher PLSD posthoc test. $P$ values indicate differences between all groups. Treatment with 2 did not result in significant improvement in colon length in DSS-treated mice (n.s.). (C) Survival of control (blue line), 4\% DSStreated (red line), DSS +2 treated (green line), and DSS +13 treated (purple line) mice.
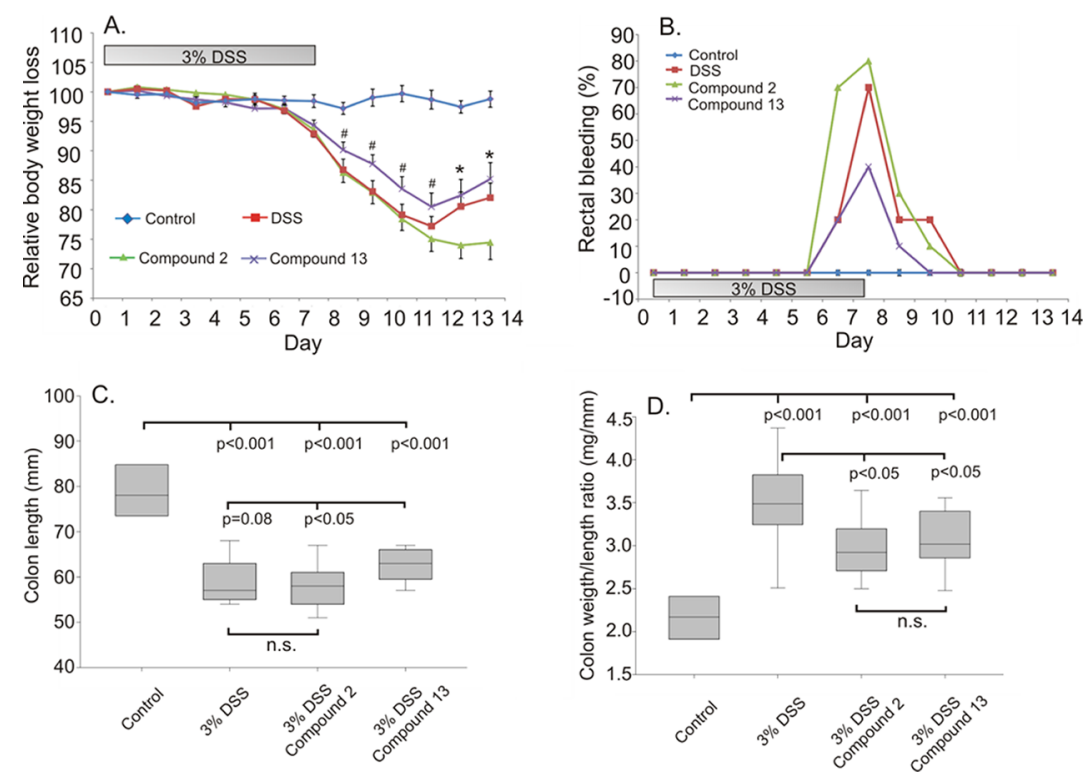

Figure 2. (A) Body weight loss in 3\% DSS colitis. Data were analyzed statistically with AVOVA followed by a Fisher PLSD posthoc test. * differences between DSS +2 and DSS + 13; statistical trend $p<0.1$. (B) Changes in the appearance of rectal bleeding expressed as a percent of mice in the respective groups. (C) Colon length in 3\% DSS colitis. Data were analyzed statistically with AVOVA followed by a Fisher PLSD posthoc test. $P$ values indicate differences between all groups. Slight, but statistically significant improvement was observed in DSS +13 compared to mice receiving DSS +2 . Treatment with 2 did not result in significant improvement in colon length in DSS-treated mice (n.s.). (D) Colon length/weight ratio in $3 \%$ DSS study. Data analyzed statistically as in panel C.

mice. Overall it appeared that 13 had a beneficial and therapeutic effect in the context of intestinal inflammation. However, an unexpectedly high mortality rate was observed among DSS-treated animals (Figure 1C). As the efficacy of DSS treatment varies by the preparation lot, the mouse strain, and the dosage, the study was repeated using $3 \%$ DSS (vide infra).

Equal mortality was observed among all treated groups in the study with $3 \%$ DSS (9 of 10 mice survived per DSS-treated group, and 10 of 10 survived in the control group). The results of the second study were consistent with the first study and demonstrated decreased morbidity and mortality for mice treated with 13 when compared to the other DSS-treated groups (Figure 2). Unexpectedly, mice treated with DSS +2 fared worse than mice treated with DSS and placebo based on body weight loss (Figure 2A). Mice treated with 13 lost significantly less weight than those receiving 2 (Figure 2A). That is, mice treated with 13 were closer to a normal body weight and, thus, were healthier. Compound 13 was also superior in terms of delaying the onset and decreasing the persistence of rectal bleeding (Figure 2B). Colon length was not a good indicator of improvement, although a positive trend was seen for mice treated with $13(p=0.08)$, but not for mice treated with 2 (Figure $2 \mathrm{C}$ ). No difference was observed in the colon weight/length ratio between the groups treated with 13 and 2 (Figure 2D).

Inflammation was measured by the release of proinflammatory cytokines from lymphoid cells of the mesenteric lymph nodes (MLNs) (Figure 3). MLN cells were collected from mice and exposed to CD3/CD28 activation ligand-bound Dynabeads in order to expand the $\mathrm{T}$ cell population. We then utilized a mouse multiplex xMAP panel designed for detection of IFN- $\gamma$, IL-17, TNF- $\alpha$, IL-1ß, IL-6, and MMP8 (neutrophil collagenase, a surrogate marker of mucosal neutrophil infiltration). Multiplex cytokine analysis showed a trend toward a better response in mice treated with 13 compared to mice treated with 2 (Figure 3A). Quantitative real-time reverse 

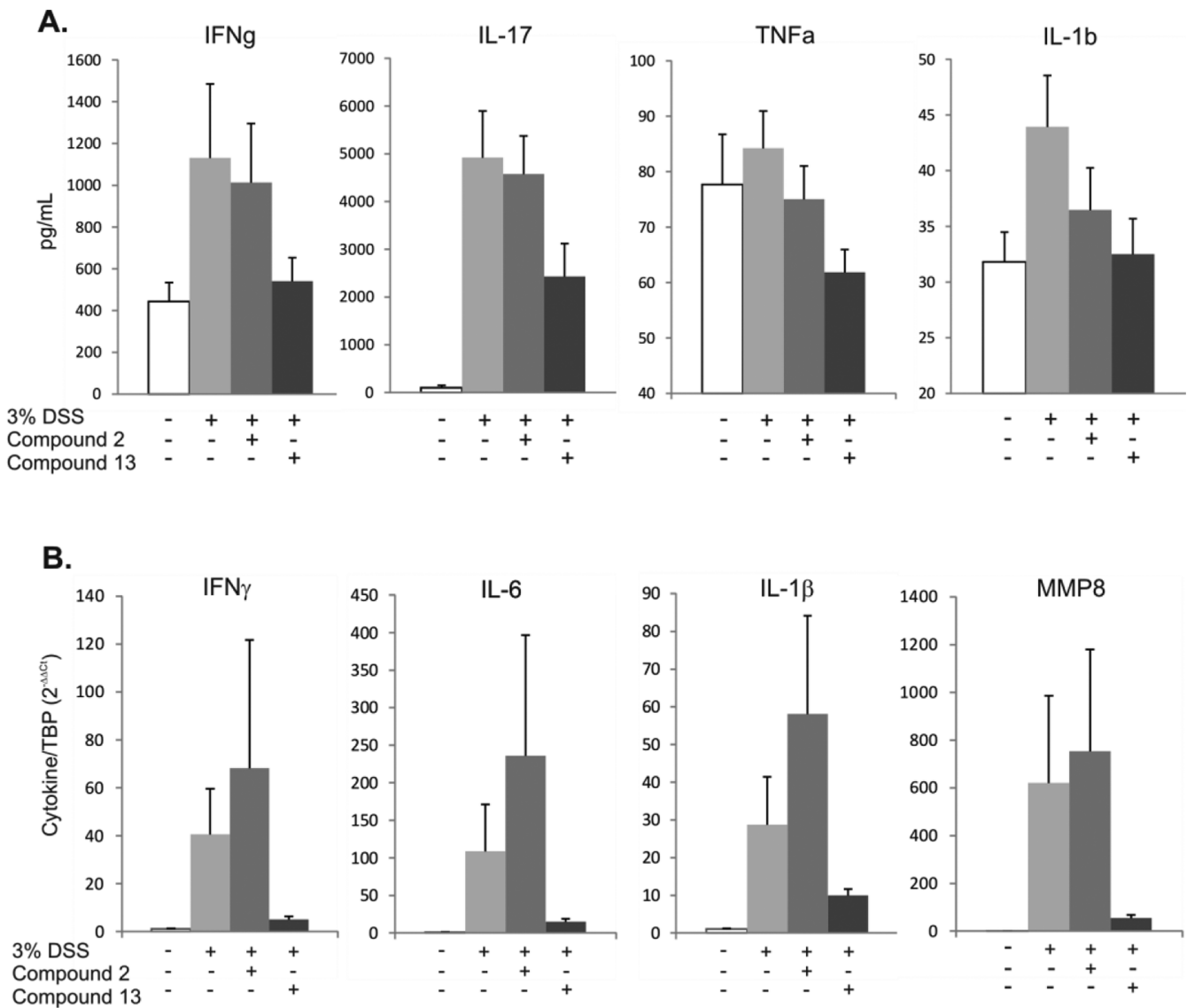

Figure 3. (A) Cytokine release from MLN cells stimulated with CD3/CD28 activation beads. (B) qRT-PCR analysis of colonic cytokine mRNA expression.

transcription PCR (qRT-PCR) analysis of colonic gene expression demonstrated generally poor and inconsistent responses to 2 . However, a consistently reduced colonic expression of IFN $\gamma$, IL-6, IL-1 $\beta$, and MMP8 was evident in mice treated with 13 (Figure 3B), suggesting that mice treated with $\mathbf{1 3}$ produced less pro-inflammatory cytokines compared to the other DSS-treated groups.

In a preliminary study of metabolism and disposition, 13 $(155.7 \mathrm{mg} /(\mathrm{kg}$ day $))$ was administered by gastric gavage to seven male 129 strain wild-type control mice and the fecal matter collected daily from the cage bedding. Feces were extracted with methanol and the extracts analyzed by high resolution ESI mass spectrometry. ${ }^{27}$ 5-ASA was detected in feces produced between 24 and $48 \mathrm{~h}$ after administration of 13 (see Supporting Information for details).

These studies with an acute in vivo model of epithelial-injury mediated colitis strongly suggest that 5-ASA pro-drug 13 may be superior to $\mathbf{2}$ and potentially to other drugs currently used for IBD treatment. We plan to evaluate the efficacy of 13 and of related constructs in more depth in follow-up studies with this and other IBD models.

\section{ASSOCIATED CONTENT}

\section{S Supporting Information}

Experimental details of the synthesis and characterization of compounds 5-9 and 11-13, copies of the ${ }^{1} \mathrm{H}$ NMR and ${ }^{13} \mathrm{C}$ NMR spectra of compounds 5-12, the ${ }^{1} \mathrm{H}$ NMR spectrum of compound 13, MALDI-TOF mass spectra of compounds 1113, and the experimental details of the bioassays and the preliminary metabolism and disposition study. This material is available free of charge via the Internet at http://pubs.acs.org.

\section{AUTHOR INFORMATION}

\section{Corresponding Author}

* P.R.K.: telephone, 520-626-9687; fax, 520-626-4141; e-mail, pkiela@peds.arizona.edu. E.A.M.: telephone, 520-621-6321; fax, 520-621-8407; e-mail, emash@email.arizona.edu.

\section{Funding}

This work was supported by Grants R33 CA 95944, RO1 CA 97360, RO1 CA 123547, and P30 CA 23074 from NCI and by RO1 DK 067286 from NIDDK.

\section{Notes}

The authors declare no competing financial interest.

\section{ABBREVIATIONS}

5-ASA, 5-aminosalicylic acid; CuAAC, copper(I)-catalyzed azide-alkyne cycloaddition; DSS, dextran sulfate sodium salt; MLN, mesenteric lymph node

\section{REFERENCES}

(1) Burger, D.; Travis, S. Conventional medical management of inflammatory bowel disease. Gastroenterology 2011, 140, 1827-1837.

(2) Williams, C.; Panaccione, R.; Ghosh, S.; Rioux, K. Optimizing clinical use of mesalazine (5-aminosalicylic acid) in inflammatory bowel disease. Ther. Adv. Gastroenterol. 2011, 4, 237-248.

(3) Sonu, I.; Lin, M. V.; Blonski, W.; Lichtenstein, G. R. Clinical pharmacology of 5-ASA compounds in inflammatory bowel disease. Gastroenterol. Clin. North Am. 2010, 39, 559-599. 
(4) Cominelli, F.; Nast, C. C.; Duchini, A.; Lee, M. Recombinant interleukin-1 receptor antagonist blocks the proinflammatory activity of endogenous interleukin-1 in rabbit immune colitis. Gastroenterology 1992, 103, 65-71.

(5) Rachmilewitz, D.; Karmeli, F.; Schwartz, L. W.; Simon, P. L. Effect of aminophenols (5-ASA and 4-ASA) on colonic interleukin-1 generation. Gut 1992, 33, 929-932.

(6) Mahida, Y. R.; Lamming, C. E.; Gallagher, A.; Hawthorne, A. B.; Hawkey, C. J. 5-Aminosalicylic acid is a potent inhibitor of interleukin 1 beta production in organ culture of colonic biopsy specimens from patients with inflammatory bowel disease. Gut 1991, 32, 50-54.

(7) Barnes, P. J.; Karin, M. Nuclear factor- $\kappa \mathrm{B}-$ a pivotal transcription factor in chronic inflammatory diseases. N. Engl. J. Med. 1997, 336, 1066-1071.

(8) Rogler, G.; Brand, K.; Vogl, D.; Page, S.; Hofmeister, R.; Andus, T.; Knuechel, R.; Baeuerle, P. A.; Schölmerich, J.; Gross, V. Nuclear factor kappaB is activated in macrophages and epithelial cells of inflamed intestinal mucosa. Gastroenterology 1998, 115, 357-369.

(9) Desreumaux, P.; Ghosh, S. Review article: mode of action and delivery of 5-aminosalicylic acid-new evidence. Aliment. Pharmacol. Ther. 2006, 24 (Suppl 1), 2-9.

(10) Safdi, A.; Pieniaszek, H.; Grigston, A.; Forbes, W. Multiple-dose pharmacokinetics of granulated mesalamine, a unique formulation providing delayed and extended release of 5-ASA. Am. J. Gastroenterol. 2008, 103, S439-S440.

(11) Wilding, I. R.; Behrens, C.; Tardif, S. J.; Wray, H.; Bias, P.; Albrecht, W. Combined scintigraphic and pharmacokinetic investigation of enteric-coated mesalazine micropellets in healthy subjects. Aliment. Pharmacol. Ther. 2003, 17, 1153-1162.

(12) Svartz, N. Salazopyrin, a new sulfanilamide preparation. Acta Med. Scand. 1942, 110, 557-598.

(13) Nielsen, O. H. Sulfasalazine intolerance. A retrospective survey of the reasons for discontinuing treatment with sulfasalazine in patients with chronic inflammatory bowel disease. Scand. J. Gastroenterol. 1982, 17, 389-393.

(14) Jandacek, R. J.; Kester, J. J.; Papa, A. J.; Wehmeier, T. J.; Lin, P. Y. T. Olestra formulation and the gastrointestinal tract. Lipids 1999, 34, 771-783.

(15) Akoh, C. C. Lipid-based synthetic fat substitutes. Food Sci. Technol. 2002, 117, 695-727.

(16) Bimal, C.; Guonong, Z. Olestra: a solution to food fat? Food Rev. Int. 2006, 22, 245-258.

(17) Jagadish, B.; Sankaranarayanan, R.; Xu, L.; Richards, R.; Vagner, J.; Hruby, V. J.; Gillies, R. J.; Mash, E. A. Squalene-derived flexible linkers for bioactive peptides. Bioorg. Med. Chem. Lett. 2007, 17, 33103313.

(18) Alleti, R.; Rao, V.; Xu, L.; Gillies, R. J.; Mash, E. A. A solanesolderived scaffold for multimerization of bioactive peptides. J. Org. Chem. 2010, 75, 5895-5903.

(19) Rao, V.; Alleti, R.; Xu, L.; Tafreshi, N. K.; Morse, D. L.; Gillies, R. J.; Mash, E. A. A sucrose-derived scaffold for multimerization of bioactive peptides. Bioorg. Med. Chem. 2011, 19, 6474-6482.

(20) Gil, M. V.; Arevalo, M. J.; Lopez, O. Click chemistry-what's in a name? Triazole synthesis and beyond. Synthesis 2007, 11, 15891620.

(21) Hein, J. E.; Fokin, V. V. Copper-catalyzed azide-alkyne cycloaddition (CuAAC) and beyond: new reactivity of copper(I) acetylides. Chem. Soc. Rev. 2010, 39, 1302-1315.

(22) Narlawar, R.; Lane, J. R.; Doddareddy, M.; Lin, J.; Brussee, J.; IJzerman, A. P. Hybrid ortho/allosteric ligands for the adenosine $A_{1}$ receptor. J. Med. Chem. 2010, 53, 3028-3037.

(23) Compound 11 was stable in 1:9 THF/aqueous $2 \mathrm{~N}$ acetic acid over a $24 \mathrm{~h}$ period; see Supporting Information.

(24) Ikeda, I.; Tomimoto, A.; Wada, K.; Fujisawa, T.; Fujita, K.; Yonemitsu, K.; Nozaki, Y.; Endo, H.; Takahashi, H.; Yoneda, M.; Inamori, M.; Kubota, K.; Saito, S.; Nagashima, Y.; Nakagama, H.; Nakajima, A. 5-Aminosalicylic acid given in the remission stage of colitis suppresses colitis-associated cancer in a mouse colitis model. Clin. Cancer Res. 2007, 13, 6527-6531.
(25) Axelsson, L.-G.; Landström, E.; Bylund-Fellenius, A.-C. Experimental colitis induced by dextran sulphate sodium in mice: beneficial effects of sulphsalazine and olsalazine. Aliment. Pharmacol. Ther. 1998, 12, 925-934.

(26) Clapper, M. L.; Gary, M. A.; Coudry, R. A.; Litwin, S.; Chang, W.-C. L.; Devarajan, K.; Lubet, R. A.; Cooper, H. S. 5-Aminosalicylic acid inhibits colitis-associated colorectal dysplasias in the mouse model of azoxymethane/dextran sulfate sodium-induced colitis. Inflammatory Bowel Dis. 2008, 14, 1341-1347.

(27) Tjørnelund, J.; Hansen, S. H. High-performance liquid chromatographic assay of 5-aminosalicylic acid (5-ASA) and its metabolites $\mathrm{N}$ - $\beta$-D-glucopyranosyl-5-ASA, $\mathrm{N}$-acetyl-5-ASA, $\mathrm{N}$-formyl5-ASA and N-butyryl-5-ASA in biological fluids. J. Chromatogr. 1991, 570, 109-117. 